Article

\title{
Tracking Positioning Algorithm for Direction of Arrival Based on Direction Lock Loop
}

\section{Xiu-Zhi Cheng ${ }^{1, *}$, Da-Rong Zhu ${ }^{1}$, Shen Zhang ${ }^{2}$ and Ping He ${ }^{1}$}

1 School of Mechanical and Electrical Engineering, Anhui Jianzhu University, Hefei 230022, Anhui, China; E-Mails: zhudarong@163.com (D.-R.Z.); heping@126.com (P.H.)

2 CUMT-IoT Perception Mine Research Center, Xuzhou 221008, Jiangsu, China; E-Mail: zhangshen@126.com

* Author to whom correspondence should be addressed; E-Mail: chengxiuzhi863@163.com; Tel.: +86-135-0569-6517; Fax: +86-0551-6382-8101.

Received: 23 January 2015 / Accepted: 8 June 2015 / Published: 26 June 2015

\begin{abstract}
In order to solve the problem of poor real-time performance, low accuracy and high computational complexity in the traditional process of locating and tracking of Direction of Arrival (DOA) of moving targets, this paper proposes a DOA algorithm based on the Direction Lock Loop (DILL) which adopts Lock Loop structure to realize the estimation and location of DOA and can adjust the direction automatically along with the changes of a signal's angular variation to track the position of the signal. Meanwhile, to reduce the influence of nonlinearity and noise on its performance, the UKF filter is designed for eliminating interference of the estimated target signal to improve accuracy of the signal tracking and stability of the system. Simulation results prove that the algorithm can not only get a high resolution DOA estimate signal, but can also locate and track multiple mobile targets effectively with enhanced accuracy, efficiency and stability.
\end{abstract}

Keywords: direction lock loop; DOA Estimate; UKF Filtering; location and tracking

\section{Introduction}

Locating and tracking of mobile targets is mainly related to observation state variables such as angles, rate of angle changes and gradient of Doppler frequency, most of which are in line with nonlinear functions. In the traditional locating and tracking of DOA, estimation algorithms based on MUSIC, ESPRIT are widely used [1,2]. However, the MUSIC algorithm needs Eigen decomposition and spectral 
peak search, rising higher computational complexity and can not satisfy the requirement, particularly when the system needs a high real-time performance [3]. ESPRIT algorithm decomposes the receipt signals into two sub-matrixes to estimate the DOA by using the phase difference between the different signals and sub-matrixes [4]. The disadvantages, including low accuracy and resolution and real-time performance, influence the communication system. To overcome this problem, Dongyoun Seo proposed a means of estimation based on DILL whose delay of synchronizing signal is similar to that of the Least Mean Square (LMS) algorithm [5]. Using the error signal to update the estimating signal by iteration achieves good performance with easier computational complexity. However, the locating and tracking performance is not very good due to the nonlinearity and noise of the system. Therefore, this paper proposes a method to enhance accurate tracking by processing the signal in smoothing on the basis of estimating the signal DOA.

There are Extended Kalman Filter (EKF) and Modified Gain EKF (MGEKF) algorithms for the processing of nonlinear system filtering [6-8]. The EKF algorithm carries linear processing of the nonlinear equation by Taylor expansion, and then it makes filter tracking by EKF. This algorithm may lead to linearization error, filtering divergence and degrading performance and is mainly used in the weak nonlinear approximated Gaussian environment. Unscented Kalman Filtering (UKF) chooses the Sigma sampling points set with mean value and same covariance based on the Unscented Transformation (UT) to estimate the posterior probability density distribution of the state vector by the statistical magnitude of sampling points after nonlinear transform. In place of the Page File (PF) random sampling by deterministic sampling, it eliminates the linear error in the EKF linearization $[9,10]$. Compared with the former two algorithms based on EKF, this algorithm enhances the convergence rate and filter tracking as well as the stability of the system. Therefore, the system can adopt the efficient UKF algorithm to do filter processing and reduce the noise interruption, decreasing the computational complexity and improving the locating accuracy and efficiency to maintain accurate tracking of the mobile targets.

\section{System Modeling}

The system is constructed on the basis of DILL structure like shown in the Figure 1. The front-end receiver carries space sampling of different DOA signals and then transforms them into baseband signals by down-conversion. The signals are spatially filtered in the filter rejector to judge, recognize the users' signals and restrain some noises. The signal received by the antenna is transmitted to the DOA estimator and used to estimate the DOA information. Finally, the estimating signals are processed by the UKF algorithm and the state distribution of the exact approximated signals is realized by recursion Bayes estimation. We can attain many approximations of the actual values, improving the update processing and tracking efficiency and accuracy to make the system more stable and efficient.

In the tracking model, the spatial signal filter is a beam space matrix made up of a baseband low-pass filter and a group of orthogonal weighing coefficients. The scene of judge original signals by the beam space matrix is shown in Figure 2, where the abscissa axis denotes the multiple orthogonal beam space confined to $0 \sim 360^{\circ}$. A half beam width of the adjacent two beams is overlapped and we call it the power beam device. We select the baseband signals by passing different power beam devices. If we can get a relatively large power output, this implies that the signal is between the two space angles formed by the 
power beam device. Then we can attain the distribution interval of the signal DOA and further can judge and recognize the distribution of the mobile signal source DOA, providing the initial data for the subsequent extract location in the DILL.

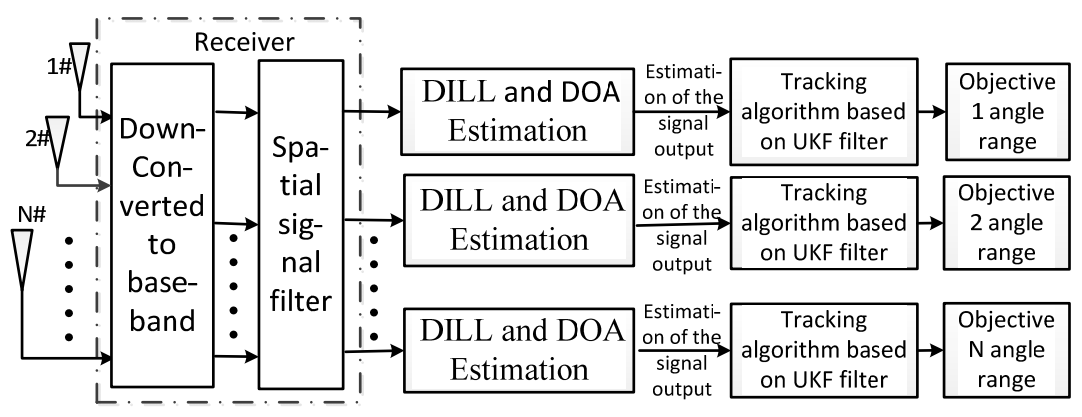

Figure 1. Locating and tracking model.

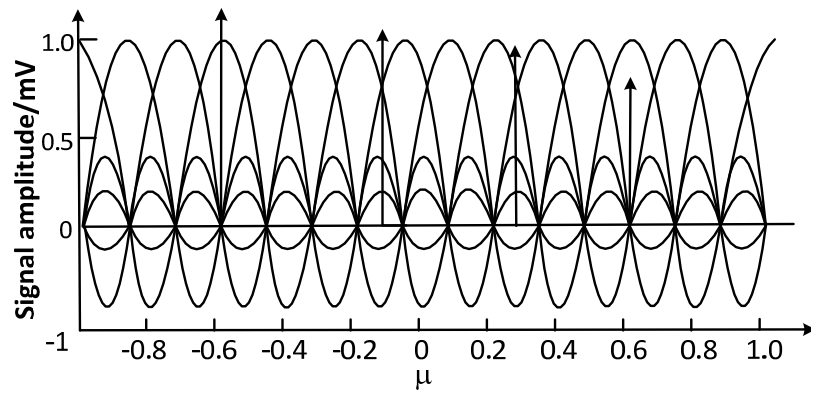

Figure 2. Beam space matrix.

The tracking device of the DILL is similar to the phase-lock loop as it is modified from the later one. Like the tracking device of the phase-lock loop shown in Figure 3, we replace the phase discriminator by the angle detector, the loop filter and digital-controlled oscillator. The angle detector is made up of two angular deflection correlators and an array vector generator to convert the related angles [11,12].

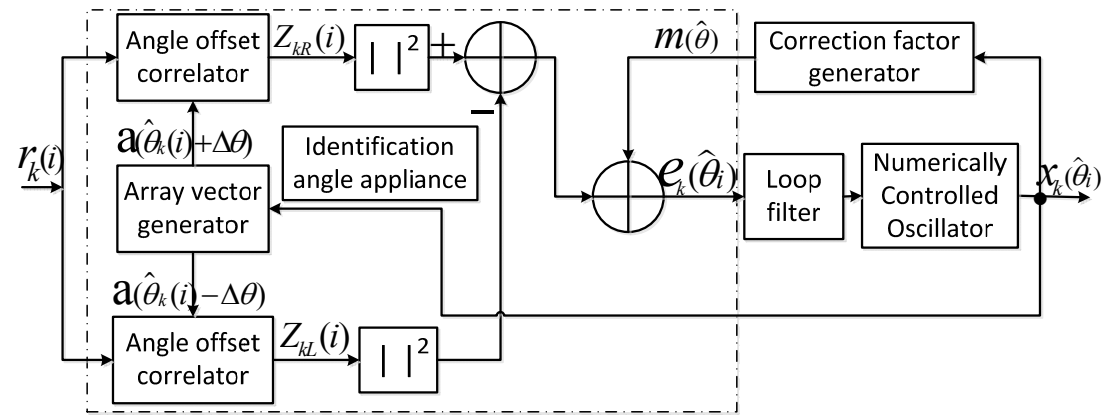

Figure 3. Tracking device in the Direction Lock Loop (DILL).

The rough estimated signal $r_{k}(i)$ is transmitted to the angular detector in the DILL, and then we carry out angular deflection related operations of the $r_{k}(i)$ and $a(\hat{\theta}(i) \pm \Delta \theta)$ produced from the array vector generator. Then we update the error signal by subtracting the square of their molds to make a more exact approximation to the actual signal. We already did a DOA rough estimation of the signal source by spatial filter in the forepart process, making the receipt signal in the pull-in range in the estimation stage. 
In the whole feedback regulation process, we transmit the $e_{k}\left(\hat{\theta}_{i}\right)$ through a loop filter to control the digital-controlled oscillator, realizing the self-adapting real-time locating and tracking in the pull-in range.

The estimated signal by the DOA algorithm is not very exact due to the various Gaussian noises in the loop output signal and $t$ the nonlinear influence on the communication system. At the same time, the number of estimated signals in the loop out is larger than the sampling number, so we should do more sampling to estimate filtering processing. The filtering based on the UKF can remove the nonlinear influence and restrain the noise efficiently, so this paper adopts the UKF filtering tracking algorithm to update the estimated signal value to get a more exact approximation of DOA.

\section{DOA Estimation Algorithm Based on the DILL}

Assume there are $K$ narrow-band signal reflected in aerial system with $N(K<N)$ array elements, the array element length is half a wavelength. The mathematical formula of the data vector transmitted in the aerial system at time $t$ is:

$$
r(t)=a(\theta(t)) d(t)+v(t)
$$

where $r(t)=\left[r_{1}(t), \cdots, r_{K}(t)\right]^{T}$ denotes the $K \times 1$ vector of receipt signal, $a(\theta)=\left[e^{j 0} e^{j \pi \sin \theta} \cdots e^{j(K-1) \pi \sin \theta}\right]$ denotes the DOA $\theta$ at time $t$ of the mobile target signal [13]. To the simplicity, set $|d(t)|^{2}=1, v(t)=\sigma^{2} I$ denotes the noise covariance vector of $K \times 1$ step, $\sigma^{2}$ is the noise covariance. Therefore, the sampled signal of the $K$ user can be expressed by:

$$
r_{k}(i)=a\left(\theta_{k}(i)\right) d(i)+v_{k}(i)
$$

If set the angular deflection produced by array vector generator is $\Delta \theta$, we then calculate the signal $r_{k}(i)$ and angular deflection signal $a\left(\hat{\theta}_{k}(i) \pm \Delta \theta\right)$ to output $Z_{k R}(i)$ and $Z_{k L}(i)$ respectively as:

$$
\begin{aligned}
& Z_{k R}(i)=\frac{1}{N} a^{H}\left(\hat{\theta}_{k}(i)+\Delta \theta\right) r_{k}(i)=d(i) R\left(\theta_{k}, \hat{\theta}_{k}(i)+\Delta \theta\right)+v_{k R}(i) \\
& Z_{k L}(i)=\frac{1}{N} a^{H}\left(\hat{\theta}_{k}(i)-\Delta \theta\right) r_{k}(i)=d(i) R\left(\theta_{k}, \hat{\theta}_{k}(i)-\Delta \theta\right)+v_{k L}(i)
\end{aligned}
$$

where $R\left(\theta_{k}, \hat{\theta}_{k}(i) \pm \Delta \theta\right)$ is spatial correlation function and is expressed as follows:

$$
R\left(\theta_{k}, \hat{\theta}_{k}(i) \pm \Delta \theta\right)=\frac{1}{N} a^{H}\left(\hat{\theta}_{k}(i) \pm \Delta \theta\right) a\left(\theta_{k}(i)\right)
$$

$v_{k R}(i)=\frac{1}{N} \mathrm{a}^{H}\left(\hat{\theta}_{k}(i)+\Delta \theta\right) v_{k}(i), \quad v_{k L}(i)=\frac{1}{N} \mathrm{a}^{H}\left(\hat{\theta}_{k}(i)-\Delta \theta\right) v_{k}(i)$ denotes the noise offset. Like the tracking device in the DILL in Figure 3, we extract the square of the molds of the signal from the correlator to get the error adjusting signal with biased estimation as:

$$
e_{k}(i)=\left|Z_{k R}(i)\right|^{2}-\left|Z_{k L}(i)\right|^{2}=G\left(\hat{\theta}_{k}(i) \mid \theta_{k}\right)+v_{k e}(i)
$$

where $G\left(\theta_{k}(\hat{i}) \mid \theta_{k}\right)$ is the angle discrimination function which determines the performance of the DOA. It can be denoted by: 


$$
G\left(\theta_{k}(i) \mid \theta_{k}\right)=\left|R\left(\theta_{k}, \hat{\theta}_{k}(i)+\Delta \theta\right)\right|^{2}-\left|R\left(\theta_{k}, \hat{\theta}_{k}(i)-\Delta \theta\right)\right|^{2}
$$

$v_{k e}(i)$ can be expressed as:

$$
\begin{aligned}
& v_{k e}(i)=\left|v_{k R}(i)\right|^{2}-\left|v_{k L}(i)\right|+ \\
& 2 \operatorname{Re}\left\{d(i)\left(R\left(\theta_{k}, \hat{\theta}_{k}(i)+\Delta \theta\right) v_{R}^{*}(i)-R\left(\theta_{k}, \hat{\theta}_{k}(i)-\Delta \theta\right) v_{L}^{*}(i)\right)\right\}
\end{aligned}
$$

To eliminate the impact of biased estimate, we subtract one correction factor $m\left(\hat{\theta}_{k}\right)$ of the error signal $e_{k}(i)$ to attain the estimated error signal, and then we filter the radio-frequency component by loop filter and transmit the low-frequency signal to the digital-controlled oscillator to update the approximation of the DOA rather than the non-relevant correction factor $m\left(\hat{\theta}_{k}\right)$. As defined as $m\left(\hat{\theta}_{k}\right)=G\left(\hat{\theta}_{k}(i) \mid \hat{\theta}_{k}(i)\right)=\left|R\left(\hat{\theta}_{k}(i), \hat{\theta}_{k}(i)+\Delta \theta\right)\right|^{2}-\left|R\left(\hat{\theta}_{k}(i), \hat{\theta}_{k}(i)-\Delta \theta\right)\right|^{2}$ in [14], we can get the modified non-relevant estimated signal as:

$$
e_{k}(i)=G\left(\hat{\theta}_{k}(i) \mid \theta_{k}\right)-m\left(\hat{\theta}_{k}\right)+v_{k e}(i)=G\left(\hat{\theta}_{k}(i) \mid \theta_{k}\right)+v_{k e}(i)
$$

where $G_{m}\left(\hat{\theta}_{k}(i) \mid \theta_{k}\right)=G\left(\hat{\theta}_{k}(i) \mid \theta_{k}(i)\right)-m\left(\hat{\theta}_{k}\right)$, the new estimate of the DOA at time of $i+1$ is

$$
\hat{\theta}_{k}(i+1)=\hat{\theta}_{k}(i)+K_{0} \bullet\left(e_{k}(i) \otimes f_{k}(i)\right)
$$

where $f_{k}(i)$ is the pulse response of the loop filter, $K_{0}$ is the gain of the digital-controlled oscillator. $\hat{\theta}_{k}$ will be closed to the actual $\theta_{k}$ infinitely in the repeatedly updates, making the evaluated error of DOA small enough to realize the location of DOA of the signal.

To analyze the performance of the DOA estimation, this paper defines the evaluated error of DOA at time of $i$ as:

$$
\varepsilon_{k}(i) \triangleq \theta_{k}-\hat{\theta}_{k}(i)
$$

By combination of (9) and (10), we can attain the evaluated error at time of $i+1$ as:

$$
\varepsilon_{k}(i+1)=\varepsilon_{k}(i)-K_{0} \cdot\left(G_{m}\left(\hat{\theta}_{k} \mid \theta_{k}\right)+v_{k e}(i)\right) \otimes f(i)
$$

As the $\hat{\theta}_{k}$ changes with the value of $\theta_{k}$ and closes to the actual direction angle $\theta_{k}$ of the signal, their actual relationship has an influence on the system error and we define the offset error as:

$$
s\left(\theta_{k}\right)=\left.\frac{\partial G\left(\hat{\theta}_{k} \mid \theta_{k}\right)}{\partial \hat{\theta}_{k}}\right|_{\hat{\theta}_{k}=\theta_{k}}
$$

In the process of tracking $\theta_{k}$ by $\hat{\theta}_{k}$, the error of estimation $\varepsilon_{k}$ decreases gradually. When $\hat{\theta}_{k} \approx \theta_{k}$, we can know $G_{m}\left(\hat{\theta}_{k} \mid \theta_{k}\right) \approx-s_{m}\left(\theta_{k}\right) \varepsilon(i)$ while $\left.s_{m}\left(\theta_{k}\right) \triangleq \frac{\partial G_{m}\left(\hat{\theta}_{k} \mid \theta_{k}\right)}{\partial \hat{\theta}_{k}}\right|_{\hat{\theta}_{k}=\theta_{k}}$ is negative. Then we can get the value of $\varepsilon_{k}(i+1)$ is: 


$$
\varepsilon_{k}(i+1)=\varepsilon_{k}(i)+K_{0} \bullet S_{m}\left(\theta_{k}\right)\left(\varepsilon_{k}(i)-\frac{v_{k e}(i)}{S_{m}\left(\theta_{k}\right)}\right) \otimes f(i)
$$

Then we do $Z$-transformation of equation (14) to attain the functional equation of DOA estimation in the domain of $Z$ :

$$
E(z)=\frac{-K_{0} s_{m}\left(\theta_{k}\right) F(z) z^{-1}}{1-\left(1+K_{0} s_{m}\left(\theta_{k}\right) F(z)\right) z^{-1}} \bullet \frac{v_{k e}(z)}{s_{m}\left(\theta_{k}\right)}
$$

where $F(z)=\sum_{i=-\infty}^{\infty} f(i) z^{-1}$ is the $Z$-transformation of the loop filter and the transfer function of the loop system is:

$$
H(z) \triangleq \frac{-K_{0} s_{m}\left(\theta_{k}\right) F(z) z^{-1}}{1-\left(1+K_{0} s_{m}\left(\theta_{k}\right) F(z)\right) z^{-1}}
$$

As the system is nonlinear that cannot adopt the model of $Z$-domain transformation in the linear system to carry state analysis, for the value of $\hat{\theta}_{k}$, we should do nonlinear filter processing to resample estimation to update the DOA data.

\section{UKF filtering processing}

In the section two, we attain the transfer function of the loop system by deduction. Since the system is non-linear, we cannot adopt linear system theory to analyze it. Furthermore, the DILL cannot eliminate the Gaussian noise effectively and has a relatively larger error to track with poor stability. In order to keep rapid, accurate tracking of mobile targets effectively, we adopt UKF filtering processing to filter the receipt signals, solving the defect of nonlinear distribution in the statistical property by using the UT transformation into Kalman filtering to attain the more accurate approximation of mean value and covariance of the signals.

After the Kth target signal source passes the DILL, the signal sampling points become more intensive as the orientation angle $\hat{\theta}_{k}$ fluctuates around the $\theta_{k}$. Set $x_{k}\left(\hat{\theta}_{i}\right)$ is sample output of Kth signal, for simplicity, this paper defines the signal as $X_{i}=x_{k}\left(\hat{\theta}_{i}\right)$ and the specific process of UKF filtering as follows:

Assume the state equation and measurement equation of the non-linear discrete system is respectively [14]:

$$
\left\{\begin{array}{l}
X_{i+1}=F\left(X_{i}, v_{i}\right) \\
Y_{i}=H\left(X_{i}, n_{i}\right)
\end{array}\right.
$$

Where $X_{i+1}, X_{i}$ is the state vector at the sampling instant of $i+1, i$ while $Y_{i}$ is observation vector, and $v_{i}, n_{i}$ is the noise vector. Then we estimate the $X_{k}$ by UKF filtering.

(a) initialize the output variable $X_{i}$ of DILL: 


$$
\begin{aligned}
& \widehat{X}_{0}=E\left\{\widehat{X}_{0}\right\} \\
& P_{0}=E\left\{\left(X_{0}-\hat{X}_{0}\right)\left(X_{0}-\hat{X}_{0}\right)^{T}\right\} \\
& \hat{X}_{i}^{a}=E\left\{X_{0}^{a}\right\}=\left[\begin{array}{lll}
\hat{X_{0}^{a}} & 0 & 0
\end{array}\right]^{T} \\
& P_{0}^{2}=E\left\{\left(X_{0}^{a}-\hat{X_{0}^{a}}\right)\left(X_{0}^{a}-\hat{X}_{0}^{a}\right)^{T}\right\}=\left[\begin{array}{ccc}
P_{0} & 0 & 0 \\
0 & P_{v} & 0 \\
0 & 0 & P_{n}
\end{array}\right]
\end{aligned}
$$

where $X^{a}=\left[\begin{array}{lll}X^{T} & v^{T} & n^{T}\end{array}\right]^{T}, P_{v}, P_{n}$ is state noise variance and measurement noise variance respectively. If $i \in\{1,2, \cdots, \infty\}$, then

(b) calculate the sampling pot value of the variable:

$$
X_{i-1}^{a}=\left[\hat{X_{i-1}^{a}} \quad \hat{X_{i-1}^{a}} \pm \sqrt{(L+1) P_{i-1}^{a}}\right]
$$

(c) estimate the state transformation:

$$
\begin{aligned}
& X_{i l i-1}^{x}=f\left[X_{i-1}^{x}, X_{i-1}^{v}\right] \\
& \hat{X}_{i}^{-}=\sum_{j=0}^{2 L} W_{i}^{(m)} X_{j, i l i-1}^{x} \\
& P_{i}=\sum_{j=0}^{2 L} W_{j}^{(c)}\left[X_{j, i l i-1}^{x}-\hat{X_{i}^{-}}\right]\left[X_{j, i l i-1}^{x}-\hat{X}_{i}^{\top}\right]^{T}+R^{\nu}
\end{aligned}
$$

where $R^{v}$ is the state noise variance of the system.

(d) estimate the measurement transformation:

$$
\begin{aligned}
& y_{i l i-1}=h\left[X_{i \mid i-1}^{x}, X_{i \mid i-1}^{n}\right] \\
& \hat{y_{i}^{-}}=\sum_{j=0}^{2 L} W_{j}^{(m)} y_{j, i l i-1} \\
& P_{y_{i} y_{i}}=\sum_{j=0}^{2 L} W_{j}^{(c)}\left[y_{j, i \mid i-1}-\hat{y_{i}^{-}}\right]\left[y_{j, i l i-1}-\hat{Y}_{i}^{-}\right]^{T}+R^{n}
\end{aligned}
$$

where $R^{n}$ is the measurement noise variance of the system.

(e) calculate the cross covariance of state and measurement estimation:

$$
P_{x_{i}, y_{i}}=\sum_{j=0}^{2 L} W_{j}^{(c)}\left[X_{j, i \mid i-1}-\hat{X}_{i}^{-}\right]\left[Y_{j, i \mid i-1}-\hat{Y}_{i}^{-}\right]^{T}+R^{v}
$$

(f) renew the weight gain and state:

$$
\begin{aligned}
& I_{i}=P_{X_{i} y_{i}} P_{y_{i} y_{i}}^{-1} \\
& \widehat{X}_{i}=\widehat{X}_{i}^{-}+I_{i}\left(Y_{i}-\hat{Y}_{i}^{-}\right)
\end{aligned}
$$

We can get the new angle predictor $\widehat{X}_{i}$ to reduce the error of non-linear estimation and restrain the noise effectively. By the above-mentioned iterative UKF filtering can we make a more accurate and reliable track of non-linear system. 


\section{Simulation results and performance comparison}

First, we make a simulation of the location and tracking of DOA estimator of DILL. We set the antenna array elements of the system as 16 with a distance of half a wave length and the SNR as $5 \mathrm{~dB}$. Assume a target signal source A entering in the antenna system with $20^{\circ}$ direction, it is then located and tracked in the DILL after reorganization by spatial filter. As shown in Figure 4, we make estimated tracking of DOA without UKF filtering, we then can know that the approximation of the DOA fluctuated around the real value while the fluctuation range is relatively large and the estimated accuracy is low due to the influence of non-linear system and noise.

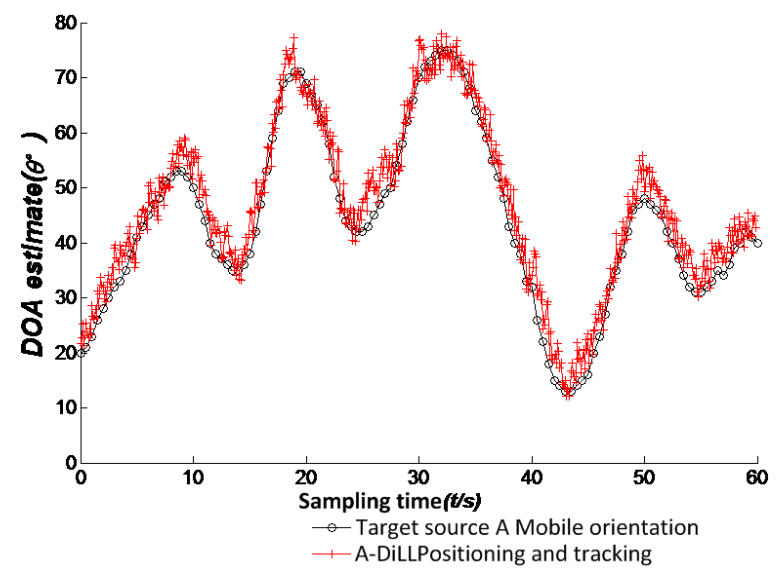

Figure 4. Estimated tracking of Direction of Arrival (DOA) in DILL.

In Figure 5, after UKF filtering, it reduces the influence of nonlinear and noise on DOA estimation, making the estimated value $\hat{\theta}_{k}$ more close to the actual value $\theta_{k}$. To analyze the effect of UKF filtering we draw the estimation error $\varepsilon_{k}$ of DOA before filtering. Just like Figure $6, \varepsilon_{k}$ fluctuates around $0^{\circ}$ and closes to $0^{\circ}$ with a relatively small range after UKF filtering, improving the DOA accuracy. When the target moves slowly, the tracking error is between $\left[-1.5^{\circ}, 1.5^{\circ}\right]$. However, the error increases and could surpass $\pm 2^{\circ}$ and the location performance decreases when the target moves faster, implying that the real-time performance of the system needs improvement.

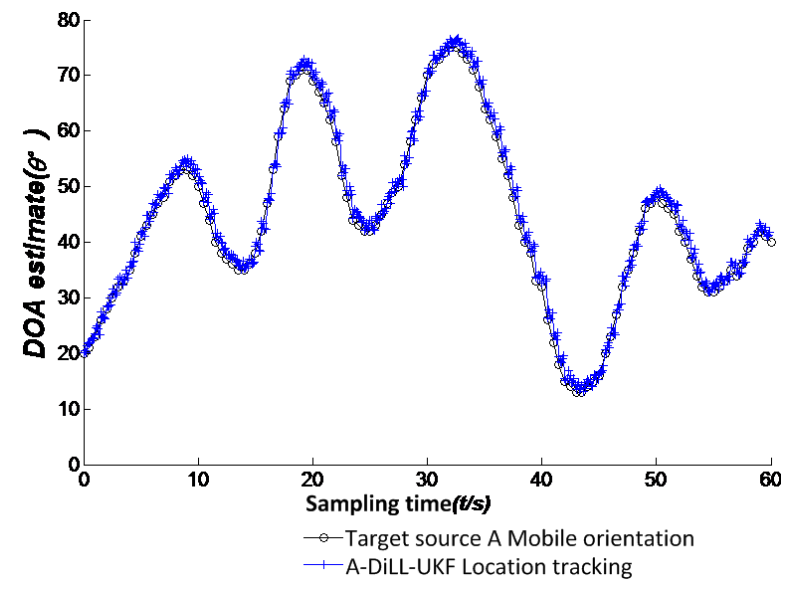

Figure 5. Location and tracking of DILL-Unscented Kalman Filtering (UKF). 


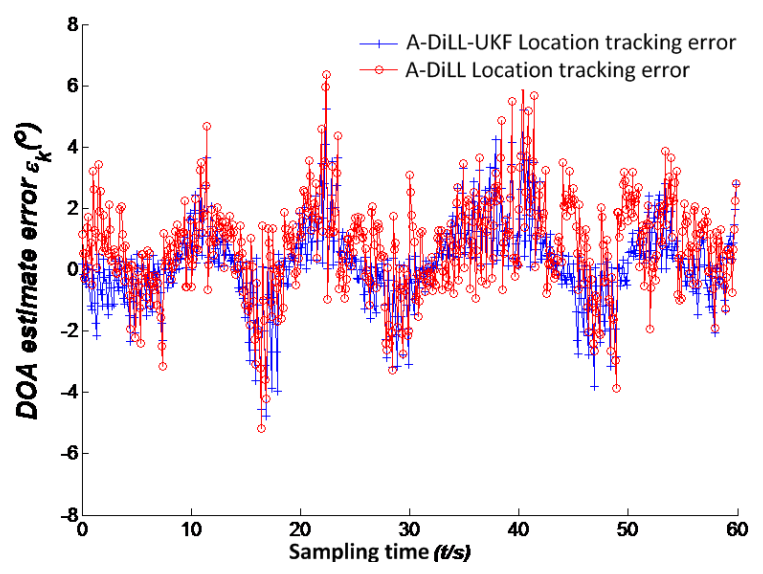

Figure 6. Estimated error of DOA.

We add another mobile signal source $B$ in the direction of $-57^{\circ}$ to do uniform circular motion with a slow angular velocity just like that shown in Figure 7. We can know that the DOA change of B is a straight line with a low angle change rate. Compared to $\mathrm{A}$, the location of $\mathrm{B}$ is much better. Therefore, the system can locate and track several targets with more accurate and reliable performance.

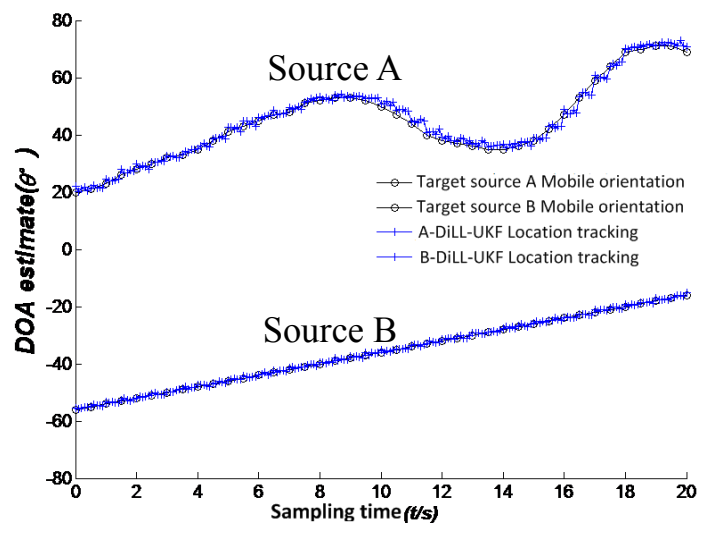

Figure 7. Location and tracking of 2 targets by DOA.

\section{Conclusions}

The DOA estimation model in DILL in this paper can not only realize the DOA estimation of a mobile target but also locate and track the targets in real time. In order to eliminate the influence of nonlinearity and noise on the system error, we choose the UKF filtering based on UT transformation to filter the DOA estimated signal to get a tracking waveform with higher accuracy. At the same time, this differs from the traditional DOA estimation algorithm and tracking models in its simple computational complexity and reliable stability. It deserves great attention in the fields of future communication systems.

\section{Acknowledgments}

We thank the support of these funds. Foundation item: National Natural Science Foundation of China (61073161); Scientific Research Fund Project (1301022066); Natural Science Fund Project of Key (KJ2014A040); Research Fund for the Doctoral Program (K02667). 


\section{Author Contributions}

Xiu-Zhi Cheng designed the concept of the article, created the system model, and wrote the main part of the paper. Da-Rong Zhu performed the literature review, Ping He contributed to system simulation, and Shen Zhang contributed to the data analysis.

\section{Conflicts of Interest}

The authors declare no conflict of interest.

\section{References}

1. Le Bihan, N.; Miron, S.; Mars, J.I. MUSIC algorithm for vector-sensors array using biquaternions. IEEE Trans. Signal Process. 2007, 55, 4523-4533.

2. Badeau, R.; Richard, G.; David, B. Performance of ESPRIT for estimating mixtures of complex exponentials modulated by polynomials. IEEE Trans. Signal Process. 2008, 56, 492-504.

3. Wei, Y.-S.; Tan, J.-B.; Guo, R. Parallel computing algorithm for MUSIC spatial spectrum estimation. Syst. Eng. Electron. 2012, 34, 12-16.

4. Zhao, X.-H.; Yang, J.; Zhao, X.-L. Research of the Increase of Smart Antenna DOA Estimate Veracity. Chin. J. Sens. Actuators 2007, 20, 132-137.

5. Seo, D.Y.; Min, S.Y.; Lee, K.B.; Lee, Y.-H. Coherent DiLL for Direction of Arrival Estimation. 2002 6th Int. Conf. Signal Process. 2002, 1, 334-337.

6. Deng, H.-B.; Zhang, L.; Wu, Y.; Zhou, J.; Liu, F. Research on track estimation based on Kalman filtering algorithm. Transducer Microsyst. Technol. 2012, 31, 4-7.

7. Song, T.L.; Speyer, J.L. A stochastic analysis of a modified gain extended Kalman filter with applications to estimation with bearings only measurements. IEEE Trans. Autom. Control 1985, 30, 940-949.

8. Zhang, P.; Huang, C.; Qiao, X. Study on SINS/GPS Deeply Integrated Navigation Based on Adaptive Extended Kalman Filter. Chin. J. Sens. Actuators 2010, 23, 408-412.

9. Liu, X.; Song, C.; Hu, L.; Zhong, Z. A Locating and Tracking Algorithm Based on UKF. J. Detect. Control 2012, 34, 71-75.

10. Julier, S.J.; Uhlmann, J.K. Unscented filtering and nonlinear estimation. Proc. IEEE 2004, 92, 401-422.

11. Min, S.Y.; Seo, D.Y.; Lee, K.B.; Kwon, H.M.; Lee, Y.-H. Direction-of-arrival tracking scheme for DS/CDMA systems: Direction lock loop. IEEE Trans. Wireless Commun. 2004, 3, 191-202.

12. Min, S.Y.; Lee, K.B.; Lee, Y.-H. Direction of arrival estimation algorithm: Direction lock loop. In Proceedings of CDMA International Conference, Seoul, Korea, 21 May 2002.

13. Liberti, J.C.; Rappaport, T.S. Smart Antenna in Wireless Communication: IS-95 and Third Generation CDMA Application, 1st ed.; Mechanical Industry Press: Beijing, China, 2002. 
14. Wang, A.-D.; Qiu, Y.-Y.; Yu, Y.-P.; Li, S.-J. Tracking algorithm for multiple spatial targets based on direction lock loop. J. Zhejiang Univ. (Eng. Sci.) 2011, 45, 1052-1056.

(C) 2015 by the authors; licensee MDPI, Basel, Switzerland. This article is an open access article distributed under the terms and conditions of the Creative Commons Attribution license (http://creativecommons.org/licenses/by/4.0/). 